Introduction Gestational diabetes (GDM) has been shown to cause adverse fetal outcomes including macrosomia, shoulder dystocia and stillbirth. The multinational Hyperglycaemia and Pregnancy Outcome (HAPO) Study has shown there is a linear relationship between oGTT result and fetal growth. Investigating epidemiological risk factors is essential to ensure appropriate highrisk groups are being screened.

Methods and Materials A retrospective case note review was undertaken of all patients with newly diagnosed GDM $(n=321)$ over a 2-year period at the West Middlesex University Hospital. The $75 \mathrm{~g}$ oGTT results were recorded with relevant demographic data including parity, age, BMI and ethnicity.

Results Asian ethnicity had the highest fasting plasma glucose $(\mu=5.49 ; n=208)$, and 2-hour plasma glucose $(\mu=9.32 ; n=208)$. One-way Anova revealed a statistically significant difference between Caucasian, Black, Asian and Oriental ethnicities with fasting glucose $(p=0.008)$ and at 2 hours $(p=0.046)$. Regression analysis revealed a significant direct association between BMI and fasting glucose $(p=0.002 ; R=0.169)$. On simple scatter plot analysis, an inverse correlation was evident between age and fasting plasma glucose level, although this was not significant on regression analysis.

Discussion This study highlights the variation in glucose tolerance between different ethnicities. It also substantiates the known relationship between BMI and fasting plasma glucose. Historically increasing age has been associated with insulin resistance and is a known risk factor for GDM. Interestingly, this study suggests that fasting glucose amongst GDM patients is inversely related to age, perhaps owing to lifestyle factors and differing perceptions amongst younger patients.

\section{PM.85 DIAGNOSING PE IN MATERNITY PATIENTS. AN AUDIT OF RADIOLOGICAL INVESTIGATIONS AND CLINICAL INTERPRETATION}

doi:10.1136/archdischild-2013-303966.166

I Newell, K Taylor, R Kelly, D Morgan. Antrim Area Hospital, Northern HSCNI, Antrim, UK

VTE is an important cause of maternal mortality but signs and symptoms are unreliable. Ionising radiation has hazards for the fetus and mother. Current American Thoracic Society guidelines recommend $\mathrm{VQ}$ scans in preference to CTPA if chest x-ray and lower limb Doppler are negative but clinical suspicion remains.

$\mathrm{VQ}$ scans are not diagnostic, results are given as a probability of $\mathrm{PE}$, i.e. none, low, intermediate or high which can be difficult for the clinician to interpret.

We undertook an audit to compare practise with guidelines and assess clinical interpretation of VQ scans. Results were compared with CTPA. All maternity patients, between 8 weeks gestation and 6 weeks post-partum, undergoing VO or CTPA were identified from a radiology database. Clinical information was obtained from notes and computerised records. Data was analysed with excel and $2 \times 2$ tables.

78 maternity patients were identified, 5 had PE identified on CTPA. Chest x-ray was performed in $92 \%$ patients and lower limb Doppler in 68\% (audit standard 100\%). VQ scans were performed in $59 \%$ women and CTPA in 56\%. 60\% patients with low/intermediate and intermediate VQ scans underwent CTPA and 2 (33\%) were found to have PE. In the 12 patients who underwent both CTPA and $\mathrm{VQ}$ scanning, the sensitivity of $\mathrm{VQ}$ was $33 \%$ and specificity was $60 \%$.

Clinicians need to be aware of the high probability of PE in patients with intermediate results, and in many cases even a low probability result should prompt consideration of CTPA

\section{PM.86 MELANOMA IN PREGNANCY: A CASE REPORT}

doi:10.1136/archdischild-2013-303966.167

R Shaunak, C Nelson-Piercy. Guys and St Thomas' NHS Foundation Trust, London, UK
A 36 year old presented at 20 weeks gestation with an abnormal mole. Biopsy revealed a non-ulcerated Stage $1 \mathrm{~B}$ malignant melanoma with $1.3 \mathrm{~mm}$ Breslow thickness and 3.5 mitosis $/ \mathrm{mm}^{2}$ (T2a). Lymphoscintigram identified an inguinal sentinel node. She underwent sentinel node biopsy (SNB), wide local excision (WLE) and exicision of the melanoma biopsy scar. She delivered a healthy female infant at 38 weeks with no placental or fetal metastasis. Follow up of the patient over the next 10 months has shown no disease recurrence.

The challenges surrounding melanoma in pregnancy lie in the timing and mode of investigation and treatment. Studies comparing localised melanoma in pregnant and non- pregnant individuals have not identified any differences in stage, tumour thickness, lymph node metastases or survival ${ }^{1}$. The management of this patient is more aggressive that that advocated in a recent case series in which WLE under local anaesthetic, with SNB postpartum is advocated for second trimester $\mathrm{T} 1 \mathrm{~b}-2 \mathrm{~b}$ patients. ${ }^{2}$ No adverse effects to the fetus have been identified following lymphoscintigram ${ }^{3}$. In conclusion, our data support the use of lymphoscintigram with SNB during pregnancy.

\section{REFERENCES}

1. O'Meara AT, Cress $R$, Xing $G$ et al, Malignant melanoma in pregnancy. A populationbased evaluation. Cancer. 2005 15; 103(6):1217-26.

2. Broer N, Buonocore S, Goldberg $\mathrm{C}$ et al, A proposal for the timing of management of patients with melanoma presenting during pregnancy. J Surg Oncol. 20121 106(1):36-40.

3. Chakera AH, Hesse B, Burak $Z$ et al, EANM-EORTC general recommendations for sentinel node diagnostics in melanoma Eur J Nucl Med. 2009 36:1713-1742.

\section{PM.87 UNPLANNED PREGNANCY IN A WOMAN WITH HUNTINGTON'S CHOREA}

doi:10.1136/archdischild-2013-303966.168

\section{N Abbott, F Soydemir. Royal Preston Hospital, Preston, UK}

A 35 year old hospital worker was diagnosed with Huntington's disease in the previous year after following a personality change.

Her main symptoms reported was that of choriform movements and anxiety which was managed with $150 \mathrm{mg}$ of Venlafaxine. Her mother also suffers from the condition. The pregnancy was unplanned and booked at 24 weeks. The couple were aware of the $50 \%$ risk of inheritance to the baby, but declined prenatal testing as they would not have undergone a termination should the result be positive. Antenatally, her choriform movements increased causing her to fall and fracture her right humerus. She required help with activities of daily living and subsequently social workers and occupational therapists were involved in her care. There was evidence of good clinical growth, although at 34 weeks gestation scan demonstrated evidence of an enlarged heart for which all investigations were normal.

The plan was to aim for a vaginal delivery, however she presented with SROM of thick meconium in early labour. She progressed to $4 \mathrm{~cm}$ dilatation, developed a bradycardia and subsequently had a CS. The apgars were normal and the patient made a straightforward post op recovery. The enlarged heart was not confirmed postnatally.

This report focuses on the issues involved in reproductive decision making and prenatal testing in parents at risk of Huntington's disease. Both deciding to abstain from having children or to pursue prenatal testing, with a $50 \%$ chance of a positive result, reflect complex problems.

\section{PM.88 SEPSIS - KEEPING AN OPEN MIND!}

doi:10.1136/archdischild-2013-303966.169

NK Vanes, G Holding, D Jeevan, S Mukherjee. University Hospitals of Coventry and Warwickshire, Coventry, UK 
We present an unusual case of sepsis in a 23 year old lady of 14 weeks gestation (para $1+1$ ) who presented with epigatric pain. Initial presentation was with bilious vomiting which became blood stained. She reported not opening bowels for 2 days however had passed flatus. There was no significant past medical history with only appendicectomy performed 5 years previously. On examination abdomen was slightly distended with tenderness in the epigastric region. Bloods were: WCC 2.5, Neut 1.5. Other bloods including amylase were normal. She was reviewed by the surgical team 'biliary colic' and an abdominal ultrasound performed, was normal. She was reviewed by medical, surgical, obstetric and gastroenterology teams and finally the diagnosis was of hyperemesis gravidarum.

She was readmitted 48 hours later with pyrexia, tachycardia and hypotension. An ultrasound was performed which demonstrated increased free fluid and an emergency laparotomy was performed. Findings were that of a perforated distal ileum. A resection of the ascending colon and terminal ileum was performed with an ileostomy. Unfortunately, 3 days post operatively she miscarried despite the fetal heart being present immediately post operatively.

During recovery she admitted that her sister in law had contracted tuberculosis and she herself had not been vaccinated. Investigations for $\mathrm{TB}$ are currently ongoing. Intra-abdominal fluid samples have confirmed the presence of ESBL.

Although an unusual presentation, this case highlights the importance of maintaining a high suspicion of sepsis. This is especially true where blood results suggest this, in the absence of other features and ongoing symptoms.

\section{PM.89 SEVERE HEADACHES IN PREGNANCY. FIRST PRESENTATION OF ARNOLD CHIARI MALFORMATION TYPE 1}

doi:10.1136/archdischild-2013-303966.170

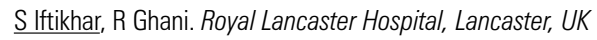

Arnold Chiari malformation is a malformation of the brain which consists of a downward displacement of the cerebellar tonsils through the foramen magnum. Type 1 Arnold Chiari is generally asymptomatic during childhood. It can typically present in women during early adulthood and can be a cause of unexplained headaches and cerebellar symptoms.

We present a case of 32 year old primigravida with severe headache and visual loss for the first time in pregnancy. She had a twin pregnancy after successful IVF. She also developed gestational diabetes. Magnetic resonance imaging of the brain showed Arnold Chiari malformation type 1 . We describe the management of this case during the antenatal and intrapartum period. She was delivered by emergency caesarean section under general anaesthetic.

Her visual loss was thought to be secondary to optic neuropathy with an unknown cause and it remains a clinical dilemma until now.

We discuss multidisciplinary team management and careful anaesthetic assessment in such cases.

\section{PM.90 INCIDENTAL PHAEOCHROMOCYTOMA IN PREGNANCY}

doi:10.1136/archdischild-2013-303966.171

BF Chen, S Al-Samarrai, M Rathi, J Rajeswary. King's Mill Hosptial, Sutton-inAshfield, UK

Introduction Phaeochromocytoma, a catecholamine-producing endocrine tumour, is a life-threatening condition to the mother and fetus. The incidence of phaeochromocytoma in pregnancy is extremely rare, 1 in 54000 pregnancies $^{1}$. If it remains undiagnosed and untreated, maternal and fetal mortality amounts to $40-50 \%^{23}$. Classically, physicians search for the tumour in hypertensive patients with paroxysmal symptoms such as headache, sweating or palpitations. However, our patient presented atypically and would have been left undiagnosed.

Case presentation A 21-year-old presented after a fall at 24 weeks gestation, with loin pain on the contralateral side to the fall. This prompted an ultrasound scan that demonstrated a $9 \mathrm{~cm}$ 'haematoma' above the right kidney. As she was claustrophobic, she declined magnetic resonance imaging (MRI). A repeat ultrasound was performed which showed the mass to be unchanged. Suspicion arose and an MRI under sedation was performed (as recommended to look for adrenal/renal mass in pregnancy ${ }^{4}$ ). This confirmed an $8 \mathrm{~cm} \times 7 \mathrm{~cm}$ mass superior to the right kidney. An endocrinologist reviewed her and serum and urine biochemistry investigations were performed.

Her urinary 3-methoxytyramine and serum dopamine levels were raised ${ }^{6}{ }^{2}$ indicating possible phaechromocytoma. She did not need $\alpha$-adrenoceptor blockade, as she remained asymptomatic throughout her pregnancy. She was referred to a tertiary hospital where an elective Caesarean section and surgical tumour removal were performed simultaneously.

Conclusion Diagnosing phaeochromocytoma in an asymptomatic pregnant patient is challenging. However, when diagnosed, a multidisciplinary team approach (obstetrician, surgeon and endocrinologist) is vital in the management of this rare disorder.

\section{REFERENCES}

1. Lenders JWM. Phaeochromocytoma and pregnancy: a deceptive connexion European Journal of Endocrinology 2012;161:143-150.

2. Schenker JG \& Chowers I. Phaeochromocytoma and pregnancy. Review of 89 cases. Obstetrical and Gynecological Survey 1971;26:739-747.

3. Dean RE. Phaeochromocytoma and pregnancy. Obstetrics and Gynecology 1958;11: $35-42$

4. Lenders JW, Eisenhofer G, Mannelli M, et al. Phaeochromocytoma. Lancet 2005;366:665-675.

5. Peleg D, Munsick RA, Diker D, Goldman JA \& Ben-Jonathan N. Distribution of catecholamines between fetal and maternal compartments during human pregnancy with emphasis on L-dopa and dopamine. Journal of Clinical Endocrinology and Metabolism 1986:62:911-914.

6. Lenders JW, Pacak K, Walther MM, Linehan WM, Mannelli M, Friberg P, Keiser HR, Goldstein DS \& Eisenhofer G. Biochemical diagnosis of phaeochromocytoma: which test is best? Journal of the American Medical Association 2002;287: 1427-1434.

\section{PM.91 HAEMATOLOGICAL INDICES IN PREGNANCY: AN IRISH TERTIARY CENTRE EXPERIENCE}

doi:10.1136/archdischild-2013-303966.172

${ }^{1} \mathrm{CM}$ McCarthy, ${ }^{2} \mathrm{MR}$ Cahill, ${ }^{1} \mathrm{~K}$ O'Donoghue. ${ }^{1}$ Department of Obstetrics and Gynaecology, Cork University Maternity Hospital, Cork, Ireland; '2Department of Haematology, Cork University Hospital, Cork, Ireland

Many haematological changes occur during pregnancy to accommodate maternal and fetal needs. Thus, monitoring of this patient groups' haematological indices are imperative. International guidelines recommend minimum haematological sampling at booking and 28 weeks.

A prospective audit was conducted between January and April 2012. Postnatal patient charts were randomly sampled, and relevant data extracted. This was entered into a secure database. Haematological indices from throughout pregnancy and within 1 week of the postnatal period were extracted from institutional laboratory systems retrospectively.

176 patients were included in our sample group, with a total of 757 samples taken. The average age of patients was 31 . Within this sample, there were 100 vaginal deliveries and 76 caesarean 